\title{
Minimum Roadside Clearance Offsets on the Inside of Reverse Curves Based on Flat Spirals
}

\author{
Timur Mauga \\ Department of Civil and Environmental Engineering, UAE University, Al-Ain, United Arab Emirates \\ Email: timur.i@uaeu.ac.ae
}

Received 8 May 2015; accepted 20 July 2015; published 24 July 2015

Copyright (C) 2015 by author and Scientific Research Publishing Inc.

This work is licensed under the Creative Commons Attribution International License (CC BY). http://creativecommons.org/licenses/by/4.0/

c) (i) Open Access

\begin{abstract}
Design guidelines require that high objects on the inside of horizontal curves be cleared so as to provide sufficient sight distance. An example of the guidelines that require such clearance is the AASHTO's Green Book. The Green Book has an analytical model for determining minimum clearance for a given design sight distance. The model is well suited for middle sections of long curves. Applying such clearance to sections near beginnings and ends of the curves and to sections where there is reverse of curvature will result in over-clearance. Over-clearance implies extra cost of earthwork where highways pass in cut zones. To avoid such extra costs the guideline suggests using the graphical method to determine exact clearance offsets. The graphical method is accurate but it is also tedious and time consuming. This study developed analytical models for efficiently determining clearance offsets that match the offsets determined with the graphical method. The offsets are ordinates from driver paths to flat roadside spirals that make the boundary of the roadside area to clear. Mathematical equations for the spirals comprise of terms related to highway speed (in the form of design sight distance), curve features, and driver locations. In turn, these terms define magnitudes of the offsets to the spirals. Combination of the terms results in many parameters to the extent of making difficult development of design charts for offsets. However, examining suitability of published offset charts for simple curves as estimates of offsets for sites with reverse curves leads to finding that the charts are suitable as long as the reverse curves have common tangents that are at least as long as $\mathbf{2 5 \%}$ of design sight distance. For reverse curves with no common tangents, offset charts have been developed and presented in this paper. Practitioners can use these charts or the derived equations to determine clearance offsets for new sites as well as for existing sites that are deficient in design sight distance.
\end{abstract}

\section{Keywords}

Reverse Curves, Sight Distance, Roadside, Clearance, offset 


\section{Introduction}

Geometric design guidelines require that sufficient sight distance be provided at all sections of highways. This requirement is automatically met on straight sections of highways since sightlines from drivers to downstream objects are within travel lanes. On curved sections, the sightlines act as chords by going through the inside of the curves. To meet the sight distance requirement on the curved sections, design guidelines recommend that the inside of the curves be cleared of high objects that block the sightlines. Each guideline has a model for determining the extent of clearance that provides design sight distances. For example, the model for determining minimum clearance offsets in the AASHTO's guideline [1] - (the Green book) is presented by Equation (1). In the equation, the design sight distance is the selected length of visibility of a highway section that allows drivers to perform specific maneuvers such as passing, stopping, or evasive maneuvers.

$$
M=R\left[1-\cos \left(\frac{28.65 \times S}{R}\right)\right]
$$

where

$M$ is the clearance offset;

$R$ is the radius of driver path;

$S$ is the design sight distance.

The model in Equation (1) was derived with consideration that both a driver and a downstream object are on horizontal curves such that the distance between the driver and the object is design sight distance. That consideration fits well sites with curves that are longer than design sight distance. Moreover, the offsets in the equation are derived as middle ordinates that bisect sightlines, making the model suitable for middle sections of curves. If these offsets are applied at other sections of long or short curves the requirement of sight distance will be met but with extra clearance. Extra clearance may imply extra earthwork and hence extra cost where highways pass through cut zones. Extra clearance may also result in unaesthetic roadside since the extra clear area could be used for decorative landscaping without negatively affecting required sight distance.

The Green Book implicitly acknowledges that offsets in Equation (1) may not be suitable for all locations. The book then suggests using two other methods for locations where designers feel Equation (1) is inapplicable. The first method is the computational method by Raymond [2]. Background mathematics of the computational method is not known but the method works for sites with isolated simple as well as spiraled curves that are longer than design sight distance. A curve is considered isolated if its curvature does not affect safety of other curves and also its safety is not affected by other curves. That condition is met where there is large spacing between curves such that a driver on one curve does not have to monitor a dangerous object on a downstream curve. On those sites, a tangent section between any two curves is at least longer than design sight distance. Where tangent sections are shorter than design sight distance the curves are not isolated and may form either compound or reverse alignmentsdepending on the direction of turning.

The second method suggested in the Green Book is the graphical method. This method works on all: isolated, compound, and reverse curves. Its drawbacks are that it is tedious and time consuming. For long, researchers have proposed substitute methods [3]-[8] that overcome these drawbacks. However, these studies focused on isolated horizontal curves and not reverse curves.

Easa [9] developed analytical models for available sight distance based on discrete objects that are located on selected locations of roadsides of reverse curves. Easa developed a table of minimum sight distance for given values of radii, radii ratios, central angles, obstacle ratios, and available clearance offsets for reverse curves with common tangents that are164 ft $(50 \mathrm{~m})$ in length. Practitioners can use the table for determining clearance offsets required to satisfy design sight distances where common tangents are $164 \mathrm{ft}$ long. More tables are needed to cover a wide range of values of common tangents, which is possible for design guidelines. Other models on available sight distance were developed by Lovell and others [10]-[12]. Further work was done by Lovell and Iida [13] who developed an algorithm that used GPS data to determine the boundary of the roadside area to clear such that design sight distance was provided. Lovell and Iida [13] called that boundary the clear zone envelope. However, no design table or design chart was developed probably due to many parameters involved.

The first objective of this paper is to develop an analytical model for efficiently determining minimum clearance offsets that would otherwise be determined with the accurate but inefficient graphical method. The offsets guarantee that available sight distances are at least equal to design sight distance on reverse curves. The second 
objective is to use the developed model to develop design charts through screening out factors that have small significance in the model. The scope of this paper is limited to reverse curves whose sum of lengths of their two circular arcs and the common tangent between the arcs is greater than design sight distance. Where the sum is shorter than design sight distance drivers have to monitor more than two downstream curves at a time, the design which may be unsafe and unaesthetic.

\section{Modeling of Analytical Offsets}

Development of mathematical equations for analytical offsets requires understanding the geometry of the roadside area to clear. The graphical method suggested by the Green Book is the basic and simplest way for describing the geometry of the roadside area. To describe the geometry, consider a reverse horizontal alignment ABCDEF shown in Figure 1(a). Point $A$ is at distance $S$ upstream of $B$ and point $F$ is at distance $S$ downstream of point $\mathrm{E}$. To determine the extent of clearance using the graphical method, a designer draws sightlines (chords) that intersect segments of the alignment. Each segment intersected has to have length that is equal to design sight distance S. The sightlines are drawn at convenient intervals, say $20 \mathrm{ft}$. The dashed lines in Figure 1(a) represent a few sightlines. After drawing the sightlines, the designer draws a roadside curve that touches all sightlines tangentially. That roadside curve is represented by the red dashed curve in Figure 1(a). The roadside curve so obtained is the boundary of the roadside area to clear. Lovell and Iida [13] called the roadside curve the clear zone envelope and hence in this paper so as to maintain consistency in the subject. In Figure 1(a), there are two envelopes, envelope 1 on the inside of curve 1 and envelope 2 on the inside of curve 2.

In Figure 1(a) it is seen that as a driver travels from point $A$ downstream, increasing is the length of a sightline from driver eyes to the point where the sightline touches the envelope tangentially. Therefore, the envelope can simply be expressed using the relationship between the length of the sightline from the eyes to the envelope

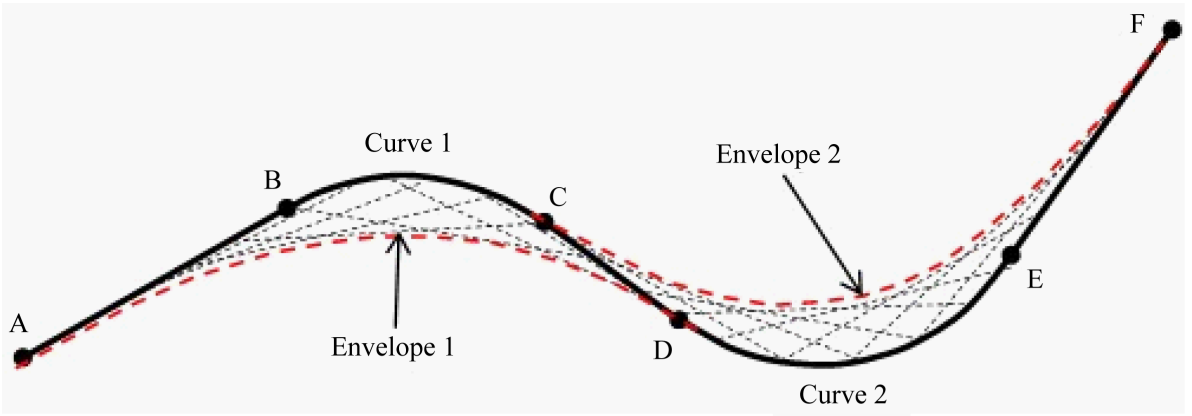

(a)

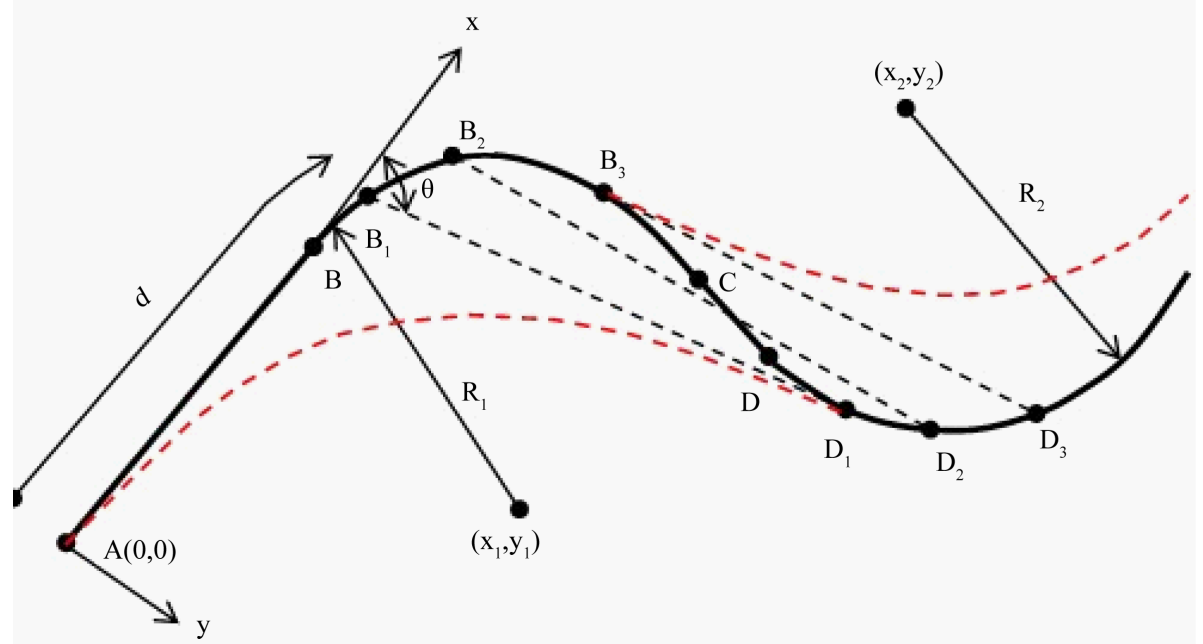

(b)

Figure 1. The graphical method and clearance envelopes. (a) Graphical method for the roadside clearance envelope; (b) Locations where sightlines are not tangents to envelopes. 
and driver location. The length of the sightline from the eyes to the tangential point on the envelope is termed here as the horizontal sightline tangent length and is denoted by $T_{H}$. Increase in $T_{H}$ can locally (using locus concepts) be described with motion of the point of tangency of the sightline and the envelope. $T_{H}$ increases as the point of tangency moves along the sightline and away from driver eyes. Combination of the motion of the point of tangency and rotation of the sightline results in the point of tangency tracing the locus that is the envelope.

Before the increase in $T_{H}$ is formulated, one consideration is made. The consideration is that, the common tangent $C D$ (in Figure 1(a) and Figure 1(b)) is shorter than sight distance thus clearance envelope 1 on the inside of curve 1 is traced as the driver travels from $A$ to $B_{1}$. Just downstream of $B_{1}$ the sightline becomes a common chord to both curve 1 and curve 2 as shown by line $B_{2} D_{2}$. When the driver reaches point $B_{3}$ the sightline is no longer a common chord and the clearance envelope 2 on the inside of curve 2 starts to trace. Sightlines for driver locations between $B_{1}$ and $B_{3}$ are not considered in derivation of equations for the envelopes since the sightlines are swinging in the area that is already clear. In other words, sightline $B_{2} D_{2}$ does not touch either envelope so $B_{2} D_{2}$ is not a critical sightline and is not a relevant sightline for determination of minimum offsets or offsets to the envelopes.

The distance from point $A$ to driver location $B_{1}$ (in Figure 1(b)) must be known before equations for envelope 1 are derived. Let $\mathrm{d}$ be the distance from $A$ to $B_{1}$ with point $A$ acting as the origin of an $\mathrm{x}-\mathrm{y}$ coordinate system. The positive sense of the $y$-axis is on the inside of curve 1 consistent with the positive sign of clearance offsets. To determine length $A B_{1}$, location of point $D_{1}$ needs to be determined first. Point $D_{1}$ is the object location corresponding to driver location $B_{1}$ and also point $D_{1}$ is where the sightline touches curve 2 tangentially before the sightline becomes a common chord to the two curves. To determine location of $D_{1}$ the mathematical equation for curves 2 is used. Equation (2) is the equation of the circular curve 2.

$$
\left(x-x_{2}\right)^{2}+\left(y-y_{2}\right)^{2}=R_{2}^{2}
$$

where

$x_{2}$ and $y_{2}$ are coordinates of the center of curve 2;

$R_{2}$ is the radius of curve 2 .

Differentiating Equation (2) with respect to $x$ yields Equation (3). Equation (3) is also for the slope at any point on curve 2.

$$
\frac{\mathrm{d} y}{\mathrm{~d} x}=-\frac{x-x_{2}}{y-y_{2}}
$$

Since point $D_{1}$ is the end of the sightline it is also the location of the downstream object. The slope of curve 2 at $D_{1}$ is found by plugging coordinates of object location in Equation (3) to yield Equation (4). Since coordinates of object location that make the sightline tangent to curve 2 are unknown, an additional equation that has the coordinates of the location of the object is needed for solving location of $D_{1}$.

$$
\frac{\mathrm{d} y}{\mathrm{~d} x}=-\frac{x_{o}-x_{2}}{y_{o}-y_{2}}
$$

where

$x_{o}$ and $y_{o}$ are object coordinates at point $D_{1}$.

The additional equation for determining location of $D_{1}$ is that of the slope of a sightline. The slope of the sightline is found by using coordinates of driver and object locations as in Equation (5). The slope of the sightline is also the trigonometric tangent of angle $\theta$ at which the sightline inclines to the x-axis.

$$
\frac{\Delta y}{\Delta x}=\tan (\theta)=\frac{y_{o}-y_{d}}{x_{o}-x_{d}}
$$

where

$x_{d}$ and $y_{d}$ are coordinates of driver location at point $B_{1}$;

$\theta$ is the angle at which a sightline inclines to the $\mathrm{x}$-axis.

Since point $D_{1}$ is where a sightline touches curve 2 tangentially, the slope of the sightline and the slope of curve 2 are equal at $D_{1}$. The equality is expressed by equating the right hand side of Equation (4) to that of Equation (5) to yield Equation (6). 


$$
-\frac{x_{o}-x_{2}}{y_{o}-y_{2}}=\frac{y_{o}-y_{d}}{x_{o}-x_{d}}
$$

To obtain coordinates of $D_{1}$ Equation (6) is solved iteratively by changing values of distance $d$ (Figure $\mathbf{1}(\mathrm{b})$ ) and using the values of $d$ to determine coordinates of driver and object locations. The solution is obtained when the sides of Equation (6) are equal. The coordinates of object location that satisfy Equation (6) are the needed coordinates for point $D_{1}$. Parallel with obtaining the coordinates is obtaining of the geometric parameters of envelope 1 at $D_{1}$. The parameters are $d_{\max }$ which is the location of the driver at $B_{1}$ (Figure 1 (b)) and $T_{H, \max }$ which is the longest sightline tangent $B_{1} D_{1}$.

\subsection{Equations for Envelope 1}

Geometric or local definition of envelope 1 is that it is a locus traced by a point (the point of tangency of a sightline to the envelope) as the point moves along the sightline and as the sightline rotates and translates. During these movements, the distance between a driver and a downstream object is fixed at the design sight distance. It is hypothesized that the motion of the point of tangency along the sightline is uniform for a given domain of driver and object locations. That is, for a given domain the horizontal sightline tangent length $T_{H}$ increases at a constant rate. An example of a domain is when a driver is on the approach tangent and an object is on curve 1 . Another example is when the drive is on curves 2 and the object is on the receding tangent. All domains are organized in two major cases according to lengths of curve 1 and curve 2. The first case is for sites with long curves and the second case is for sites with short curves. Under these two cases there are sub-cases as presented in the subsections below.

\subsubsection{Case 1: Long Curves}

This section presents derivation of envelope curves for sites with components of reverse curves that are longer than design sight distance. There are four sub-cases considered for each component curve. The first sub-case is when a driver is on the approach tangent (like point $B$ in Figure 2) and an object is on curve 1 . The second subcase is for both a driver and an object being on curve 1. The third is when a driver is on curve 1 and an object is on the common tangent. The fourth sub-case is for a driver on curve 1 (like point $C$ in Figure 2) and an object on curve 2. Below is the presentation of the sub-cases starting with sub-case 1(a).

\section{1) Case 1 (a): A driver on approach tangent, an object on curve 1}

The sightline tangent length $T_{H}$ in Figure 2 increases as $d$ (location of driver $B$ downstream of $P C_{1}-S$ ) increases. Equation (7) is for the linear relationship between $T_{H}$ and $d$. The domain of the equation is $P C_{1}-S \leq$ $d \leq P C_{1}$ or $0 \leq d \leq S$. The linear coefficient $C_{1}$ is obtained by inserting in Equation (7) known values of $T_{H}$ and $d$. The values are $d=S$ (i.e. the driver is at $P C_{1}$ ) and $T_{H}=T_{M}$ (as in Figure 2). $T_{M}$ is the length of $T_{H}$ corresponding to the offset presented earlier by Equation (1).

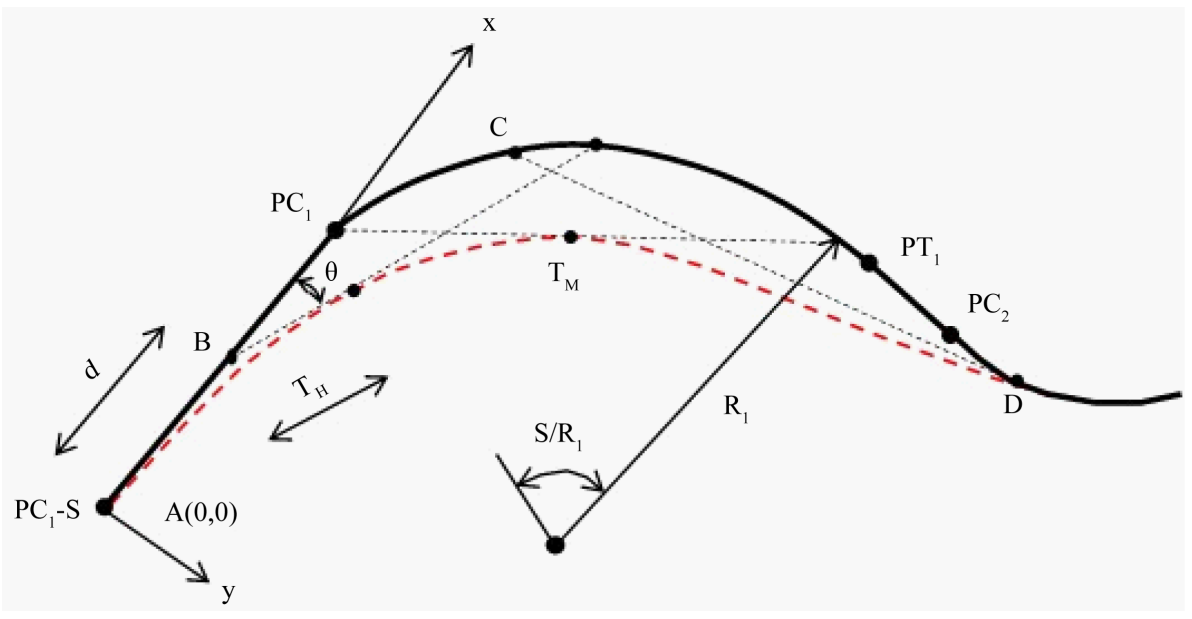

Figure 2. Formulation of envelope 1 for long curves. 


$$
T_{H}=C_{1} \cdot d
$$

where

$T_{H}$ is the distance from driver eyes to the envelope;

$d$ is the location of driver downstream of $P C_{1}-S$;

$C_{1}$ is the constant rate of change of $T_{H}$;

$C_{1}=\frac{T_{M}}{S}$; and $T_{M}=R_{1} \cdot \sin \left(\frac{28.65 \times S}{R_{1}}\right)$.

The part of envelope 1 represented by Equation (7) is a spiral since its radius decreases gradually from infinity at $P C_{1}-S$ to $R_{1}-M$ (where $M$ is given by Equation (1)) at $P C_{1}+0.5 S$. This spiral is different from, and flatter than the Euler's spiral used for transitioning turning. The differences are presented in detail in [14].

2) Case 1 (b): A driver and an object on curve 1

When both a driver and an object are on curve 1 the distance from driver eyes to the point the sightline touches the envelope tangentially is half the length of the sightline. It is half the length of the sightline since the offset (given by Equation (1)) from driver path to the sightline bisects the sightline. The formula for $T_{H}$ in this sub-case is given by Equation (8). The domain for this case is $P C_{1} \leq d \leq P T_{1}-S$. It is worth noting that constantness of $T_{H}$ in Equation (8) will yield constant offsets that are equal to the values given by Equation (1). Constantness of the offsets also implies that the part of the envelope in this sub-case is a circle of radius $R_{1}-M$.

$$
T_{H}=T_{M}
$$

where

$T_{M}$ is the length of the sightline to offset $M$ in Equation (1);

$T_{M}=R_{1} \cdot \sin \left(\frac{28.65 \times S}{R_{1}}\right)=C_{1} S$.

\section{3) Case 1 (c): A driver on curve 1, an object on common tangent}

The length of $T_{H}$ starts to increase again after the driver passes station $P T_{1}-S$. Equation (9) is for the spiral envelope in this sub-case and its domain is $P T_{1}-S \leq d \leq P T_{1}-S+T_{c}$ or $L_{1} \leq d \leq L_{1}+T_{c}$ since station $P T_{1}$ is the same as $S+L_{1}$. The first term of Equation (9) was obtained from the previous sub-case above and it serves as the initial value of $T_{H}$ within this sub-case. The constant $C_{2}$ is obtained by inserting $d=S+L_{1}$ and $T_{H}=S$ since $T_{H}=S$ covers all value of $T_{c}$ within the range $0 \leq T_{c} \leq S$.

$$
T_{H}=T_{M}+C_{2}\left(d-L_{1}\right)
$$

where

$C_{2}=\frac{S-T_{M}}{S}$.

\section{4) Case 1 (d): A driver on curve 1, an object on curve 2}

In this sub-case $T_{H}$ starts with the value obtained by inserting in Equation (9) the upper limit of its domain i.e. $d=L_{1}+T_{c} . T_{H}$ then increases until it reaches its maximum value $T_{H, \text { max }}$ which is also length $C D$ in Figure 2. The local (that of locus) meaning of $T_{H, \max }$ is the maximum value of $T_{H}$ obtained when the tangent point has moved such that it has caught up the object at point $D$ in Figure 2. The point catching up the object at $D$ implies that envelope 1 has been completely traced by the point. Point $C$ (in Figure 2) is the driver location corresponding to $T_{H \text {,max }}$; point $C$ is at distance $d=d_{\max }$ from $P C_{1}-S$. Equation (10) below is for the envelope in this subcase. The domain of the equation is $P T_{1}-S+T_{c} \leq d \leq d_{\max }$ or $L_{1}+T_{c} \leq d \leq d_{\max }$. The constant $C_{3}$ is obtained by inserting $d=d_{\max }$, and $T_{H}=T_{H, \max }$ in Equation (10).

$$
T_{H}=T_{M}+C_{2} T_{c}+C_{3}\left(d-L-T_{c}\right)
$$

where

$C_{3}=\frac{T_{H, \text { max }}-\left(T_{M}+C_{2} T_{c}\right)}{d_{\text {max }}-L-T_{c}}$ 
Equations (7) to (10) are grouped together in one equation with four regimes as given in Equation (11).

$$
T_{M}=\left\{\begin{array}{lc}
C_{1} \cdot d, & d \leq S \\
T_{M}, & S \leq d \leq L_{1} \\
T_{M}+C_{2}(d-L), & L_{1} \leq d \leq L_{1}+T_{c} \\
T_{M}+C_{2} T_{c}+C_{3}\left(d-L-T_{c}\right), & L_{1}+T_{c} \leq d \leq d_{\text {max }}
\end{array}\right.
$$

This equation is complemented by the equation for the angle at which a sightline inclines to the approach tangent. The equation for that angle was presented earlier as Equation (5).

\subsubsection{Case 2: Short Curves}

On sites where components of reverse curves are shorter than design sight distance, derivation of envelope equations is similar to case 1 but with a few differences. One of the differences is that case 2 has one more sub- case: that of driver and object being on tangents. Another difference is that segments of the envelope are all spirals, no circular part as in case 1(b). Below is presentation of derivation of equations for those spiral segments.

1) Case 2(a): A driver on approach tangent, an object on curve 1

On sites with lengths of curve 1 that are shorter than design sight distance the first regime of Equation (11) above is applicable but with a narrower domain. The domain is $P C_{1}-S \leq d \leq L_{1}$ or $0 \leq d \leq L_{1}$ (where $L_{1}$ is the length of curve 1). The domain is narrower since $L_{1}$ is shorter than $S$. Equation (12) is for this sub-case as a replica of the first regime of Equation (11).

$$
T_{H}=C_{1} \cdot d
$$

\section{2) Case 2(b): A driver on approach tangent, an object on common tangent}

Equation (13) is for this sub-case and its domain is $L_{1} \leq d \leq L_{1}+T_{c}$ for short common tangents and $L_{1} \leq d \leq$ $S$ for long common tangents. The first term of Equation (13) is from case 2(a) after setting $d=L_{1}$ in Equation (12). The resulting value is maximum for case 2(a) but minimum for case 2(b). The coefficient $C_{4}$ is obtained by setting $d=d_{M}$ and $T_{H}=T_{M}$ in Equation (13), where $d_{M}$ is the driver location corresponding to the middle offset $M$ at the middle of the curve (station $\left.P C_{1}+0.5 L_{1}\right) . T_{M}$ is half the length of the sightline since the middle offset at $P C_{1}+0.5 L_{1}$ bisects the sightline as shown in Figure 3.

$$
T_{H}=C_{1} L_{1}+C_{4}\left(d-L_{1}\right)
$$

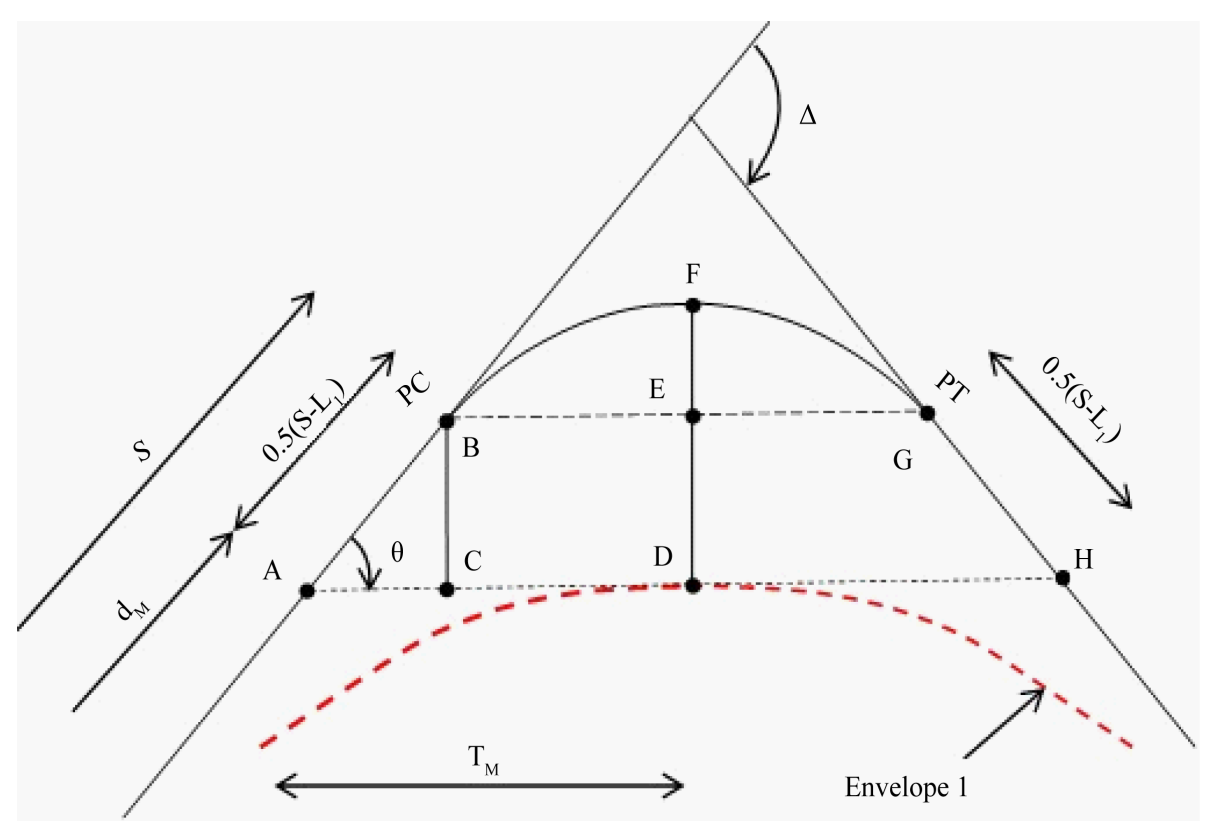

Figure 3. Middle offset $\mathrm{M}$ and tangent length $T_{M}$. 
where

$C_{4}=\frac{T_{M}-C_{1} L_{1}}{d_{M}-L_{1}} ;$

$d_{M}$ is the driver location corresponding to middle offset $M$;

$T_{M}$ is the value of $T_{H}$ corresponding to $M$.

The driver location $d_{M}$ in the formula for $C_{4}$ in Equation (13) above is given by Equation (14).

$$
d_{M}=S-0.5\left(S-L_{1}\right)
$$

The sightline tangent $T_{M}$ in $C_{4}$ is derived using Figure 3 as shown below.

$$
\begin{aligned}
& T_{M}=A C+C D=A C+B E \\
& T_{M}=A B \cdot \cos (\theta)+B E \\
& T_{M}=0.5\left(S-L_{1}\right) \cdot \cos (\theta)+R \cdot \sin (\theta)
\end{aligned}
$$

where

$\theta=0.5 \Delta$.

\section{3) Case 2(c): A driver on approach tangent, an object on curve 2}

This sub-case is relevant on sites where lengths of common tangents are shorter than $S-L_{1}$ including sites with no common tangents. Equation (18) is for this sub-case and its domain is $L_{1}+T_{c} \leq d \leq d_{\max }$ if $d_{\max } \leq S$. If $d_{\max } \leq S$ tracing of envelope 1 is completed before a driver reaches $P C_{1}$ otherwise the domain is $L_{1}+T_{c} \leq d \leq S$. The first two terms of Equation (18) are from case 2(b) after setting $d=L_{1}+T_{c}$ in Equation (13). The coefficient $C_{5}$ is obtained by setting $d=d_{\max }$ and $T_{H}=T_{H, \max }$ in Equation (18). The value of $d_{\max }$ is the location of a driver corresponding to the maximum length of sightline tangent to envelope 1 . After $d=d_{\max }$ the sightline becomes a common chord to both curve 1 and curve 2 .

$$
T_{H}=C_{1} L_{1}+C_{4} T_{c}+C_{5}\left(d-L_{1}-T_{c}\right)
$$

where

$C_{5}=\frac{T_{H, \max }-\left(C_{1} L_{1}+C_{4} T_{c}\right)}{d_{\text {max }}-L_{1}-T_{c}}$

\section{4) Case 2(d): A driver on curve 1, an object on common tangent}

This sub-case is for sites with lengths of common tangents that are longer than $S-L_{1}$. Equation (19) is for this sub-case and its domain is $S \leq d \leq L_{1}+T_{c}$. The first two terms of Equation (19) are from case 2(b) if $d=S$ is inserted in Equation (13). The coefficient $C_{6}$ is obtained by setting $d=S+L_{1}$ i.e. driver is at $P T_{1}$ and $T_{H}=S$ in Equation (19). $T_{H}=S$ covers all value of $T_{c}$ within the range $0 \leq T_{c} \leq S$.

$$
T_{H}=C_{1} L_{1}+C_{4}\left(S-L_{1}\right)+C_{6}(d-S)
$$

where

$C_{6}=\frac{S-\left[C_{1} L_{1}+C_{4}\left(S-L_{1}\right)\right]}{L_{1}}$

\section{5) Case 2(e): A driver on curve 1, an object on curve 2}

This sub-case is for both sites that have common tangents that are shorter than $S-L_{1}$ and those with common tangents that are longer than $S-L_{1}$. For former sites the relationship for sightline length is given by Equation (20). The domain for Equation (20) is $S \leq d \leq d_{\max }$. The first three terms of Equation (20) are obtained by setting $d=S$ in Equation (18). For sites with common tangents that are longer than $S-L_{1}$ Equation (21) is used. The domain for Equation (21) is $L_{1}+T_{c} \leq d \leq d_{\max }$. The first three terms of Equation (21) are from case 2(d) when $d=L_{1}+T_{c}$ in Equation (19). The coefficient $C_{7}$ in (20) and (21) is obtained by setting $d=d_{\max }$ and setting $T_{H}=T_{H \text {,max }}$ in the respective equations.

$$
T_{H}=C_{1} L_{1}+C_{4} T_{c}+C_{5}\left(S-L_{1}-T_{c}\right)+C_{7}(d-S)
$$


where

$$
\begin{aligned}
C_{7}=\frac{T_{H, \text { max }}-\left[C_{1} L_{1}+C_{4} T_{c}+C_{5}\left(S-L_{1}-T_{c}\right)\right]}{d_{\text {max }}-S} \\
T_{H}=C_{1} L_{1}+C_{4}\left(S-L_{1}\right)+C_{6}\left(L_{1}+T_{c}-S\right)+C_{7}\left(d-L_{1}-T_{c}\right)
\end{aligned}
$$

where

$$
C_{7}=\frac{T_{H, \text { max }}-\left[C_{1} L_{1}+C_{4}\left(S-L_{1}\right)+C_{6}\left(L_{1}+T_{c}-S\right)\right]}{d_{\text {max }}-L_{1}-T_{c}}
$$

\subsection{Equations for Envelope 2}

Equations for envelope 2 are also derived by tracking increases of $T_{H}$ for each case. The easiest way is by starting from $P T_{2}+S$ and moving towards $P C_{1}-S$. That way will end up with equations like those presented above. Due to limitation of space, it is avoided to duplicate the effort through re-derivation of the equations.

\subsection{Coordinates of Envelope Curves}

Equations for $T_{H}$ and $\theta$ represent the envelopes in polar like form. The equations can be used to determine rectangular coordinates along the envelopes. Equation (22) and (23) use $T_{H}$ and angle $\theta$ for determining the coordinates.

$$
\begin{aligned}
& x=x_{d}+T_{s} \cdot \cos (\theta) \\
& y=y_{d}+T_{s} \cdot \sin (\theta)
\end{aligned}
$$

\subsection{Clearance Offsets}

Offsets are normal ordinates from driver path to clearance envelopes. For the section from $P C_{1}-S$ to $P C_{1}$, like point $B$ in Figure 4, the offsets are simply y-coordinates given by Equation (23) above. For any location on curve 1 , an offset is given as radius minus length $D E$ (in Figure 4). Point $D$ has coordinates $D(x, y)$ while the center of the highway curve 1 is $E(S, R)$. Equation (24) is for the offset from the curved driver path to envelope 1.

$$
\overline{C D}=R_{1}-\overline{D E}=R_{1}-\sqrt{(x-S)^{2}+\left(y-R_{1}\right)^{2}}
$$

For a point on the common tangent between $P T_{1}$ and $P C_{2}$, say point $G$, offset $F G$ is given by the radius minus projection of line $E F$ on the radius at $P T_{1}$. Equation (25) is for that offset.

$$
\overline{F G}=R_{1}-\overline{E F} \cdot \cos (\beta-\Delta)=R_{1}-\sqrt{(x-S)^{2}+\left(y-R_{1}\right)^{2}} \cdot \cos (\beta-\Delta)
$$

For a point on curve 2, like point $I$, offset $H I$ is given by the distance from $H(x, y)$ to the center of curve 2 minus the radius $R_{2}$. Equation (26) presents offset $H I$.

$$
\overline{H I}=\overline{H J}-\overline{I J}=\sqrt{\left(x-x_{2}\right)^{2}+\left(y-y_{2}\right)^{2}}-R_{2}
$$

Equations (24) to (26) are grouped together as regimes of one equation in Equation (27).

Envelope 2 uses the same Equation(27) if offsets are determined from $P T_{2}+S$ considering the driver in the opposite direction (i.e. traveling from $P T_{2}+S$ to $P C_{1}-S$ ).

$$
m=\left\{\begin{array}{lc}
y, & X \leq P C_{1} \\
R_{1}-\sqrt{(x-S)^{2}+\left(y-R_{1}\right)^{2}}, & P C_{1}<X \leq P T_{1} \\
R_{1}-\sqrt{(x-S)^{2}+\left(y-R_{1}\right)^{2}} \cdot \cos (\beta-\Delta), & P T_{1}<X \leq P C_{2} \\
\sqrt{\left(x-x_{2}\right)^{2}+\left(y-y_{2}\right)^{2}}-R_{2}, & P C_{2} \leq X
\end{array}\right.
$$




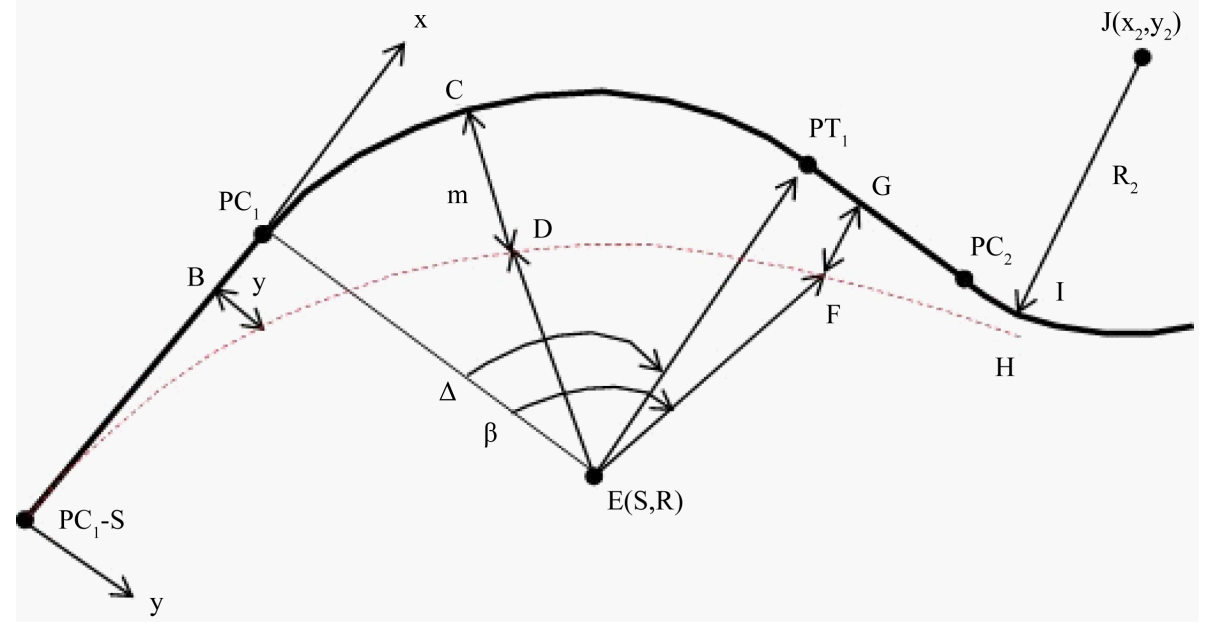

Figure 4. Relationship between offset and location.

where

$X$ is station of an offset; $m$ is an offset.

\section{Verification}

Equations for envelopes and offsets are verified using the graphical method since the equations aim at efficiently reproducing offsets determined with the graphical method. The graphical method is recommended in the Green Book as the general method for determining offsets. Offsets determined with the graphical method are geometrically sound and have been accepted by researchers and practitioners. It is known in practice that the graphical method is tedious and time consuming but its value makes it worth to use one time to validate analytical models. Consider a two lane highway with design speed of $50 \mathrm{mph}$. The design sight distance on this highway is the stopping sight distance of $425 \mathrm{ft}$. One of reverse curves on the highway has curve 1 with length of $170 \mathrm{ft}$ and radius $R_{1}=650 \mathrm{ft}$. Curve 2 has a length of $255 \mathrm{ft}$ and radius of $R_{2}=700 \mathrm{ft}$. The common tangent $T_{c}$ between $P T_{1}$ and $P C_{2}$ is $200 \mathrm{ft}$. Figure 5(a) presents lines for offsets determined with analytical equations and offsets determined with the graphical method. All offsets in the figure use one driver path as reference line. The lines for analytical offsets match well the accurate offsets produced with the graphical method. Therefore, the analytical offsets are sound. That is, the hypothesis that $T_{H}$ increases at uniform rate is practically accurate for each case considered. Unknown exact and complex equations for the envelopes may show some nonlinearity in the future but that nonlinearity is ignored without serious errors in offsets.

Presentation of offset lines in Figure 5(a) may be wrongly perceived as offsets on one side of a highway since the offset lines are on the same side of the horizontal axis. Such presentation may work well for offsets on compound curves. To avoid that wrong perception, the curves have been re-presented in Figure 5(b) to emphasize reverse of curvature. Figure 5(b) hence reflects clearance on the inside of each circular arc. For the rest of the paper, offset curves will be presented in the style of those in Figure 5(b).

\section{Design Charts}

Derived equations for envelopes and offsets have many variables. Offsets to envelope 1 are a function of $S, R_{1}$, $L_{1}, T_{c}, R_{2}$, and $L_{2}$. The factor that determines the extent to which offsets to envelope 1 depend on $R_{2}$ and $L_{2}$ is $T_{\mathrm{c}}$. There is maximum dependency when $T_{c}=0$ and no dependency when $T_{c} \geq S$. It is therefore difficult to produce a 2D design chart or table with these seven factors. However, the offset curves in Figure 5 above are close to symmetrical curves. Mauga [14] developed one design chart for simple curves and the chart has offset lines that are symmetrical about station $P C+0.5 \mathrm{~L}$. It is worth examining how much the offset chart for simple curves may be applicable to reverse curves before embarking in the task of combining numerous variables of reverse curves. Figure 6 presents graphs for the examination.

Figure 6(a) presents offset lines for a $50 \mathrm{mph}$ two-lane highway reverse curve and for two simple curves. The 


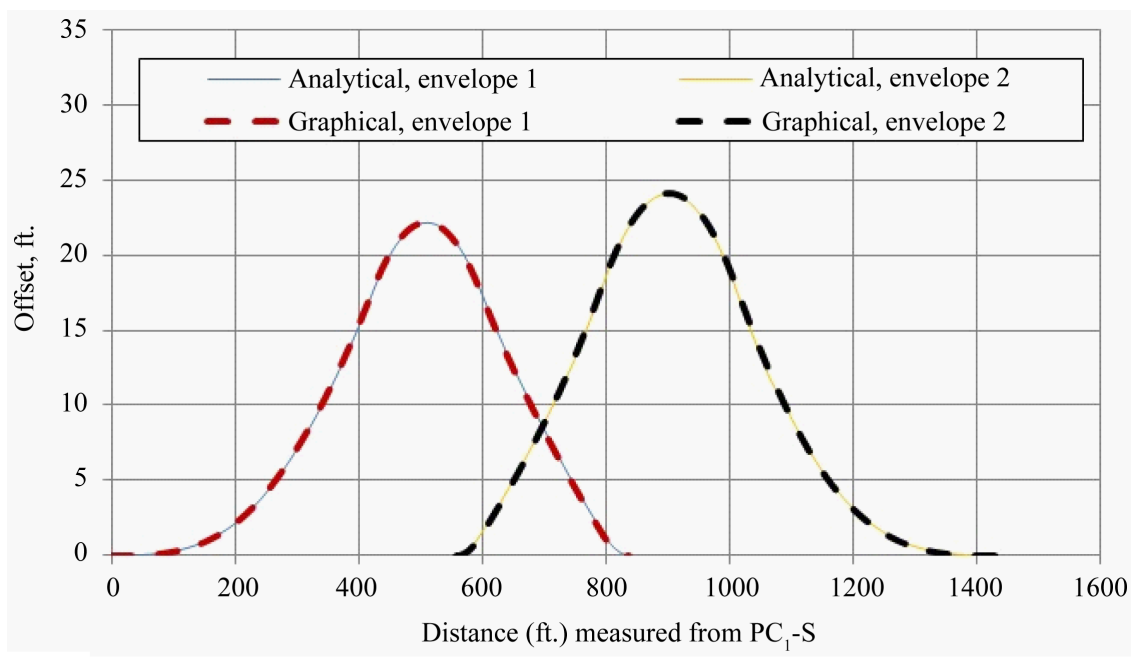

(a)

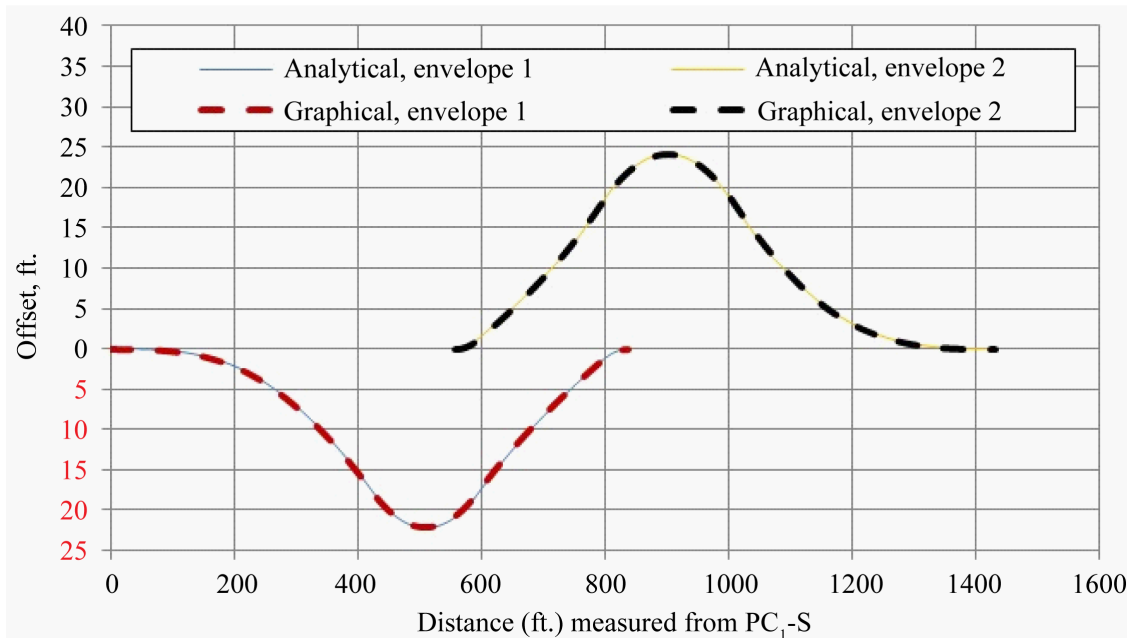

(b)

Figure 5. Verification of analytical offsets. (a) Curves of horizontal clearance offsets; (b) Offset curves presented in emphasis of reverse curvature.

offsets for simple curves were obtained by lengthening the common tangent to $S$. For a driver travelling from $P C_{1}-S$ the reverse curve has the following characteristics: $R_{1}=800 \mathrm{ft}, L_{1}=255 \mathrm{ft}, T_{C}=0, R_{2}=660 \mathrm{ft}, L_{2}=$ $170 \mathrm{ft}$, and lane width of $12 \mathrm{ft}$. The offset lines in Figure 6(a) have been coincided at $P C+0.5 \mathrm{~L}$ for each travel direction. Offsets to envelope 2 were produced by considering a driver moving from $P T_{2}+S$ to $P C_{1}-S$. Figure 6(a) shows that for a reverse curve without a common tangent, use of offset charts for simple curves will conservatively clear more than required area near the point of reverse curvature. Over-clearance guarantees that available sight distance is greater than design sight distance but with extra cost in cut zones. The fact that offsets for simple curves are greater than offsets needed for reverse curves was also reported by Easa [9]. Pure reverse curves need smaller offsets since their envelopes have smaller curvature due to sightlines reversing direction of rotation sooner than on simple curves or reverse curves with long common tangents. It is noteworthy that reversal of curvature without a common tangent is not recommended in the Green Book due to the problem of transition of superelevation and other related problems.

Increasing the value of $T_{c}$ while keeping other geometric features constant yields different results. Figure 6(b) is for the example in Figure 6(a) but with $T_{c}=106.25 \mathrm{ft}$ which is $25 \%$ of the design sight distance. It is seen that discrepancy between the offset lines has decreased tremendously near the maximum offsets. Addition of the common tangent has removed big differences in the offsets. Where there is still difference between the offset lines, first, the maximum difference is approximately $5 \mathrm{ft}$, second, the $5 \mathrm{ft}$ difference is an overestimation as op- 


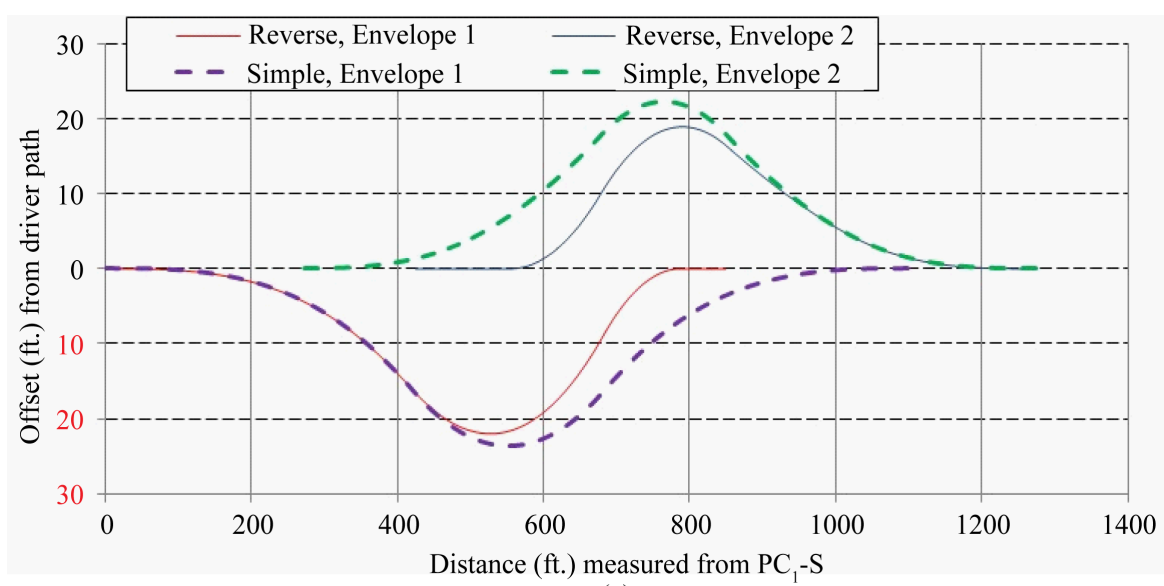

(a)

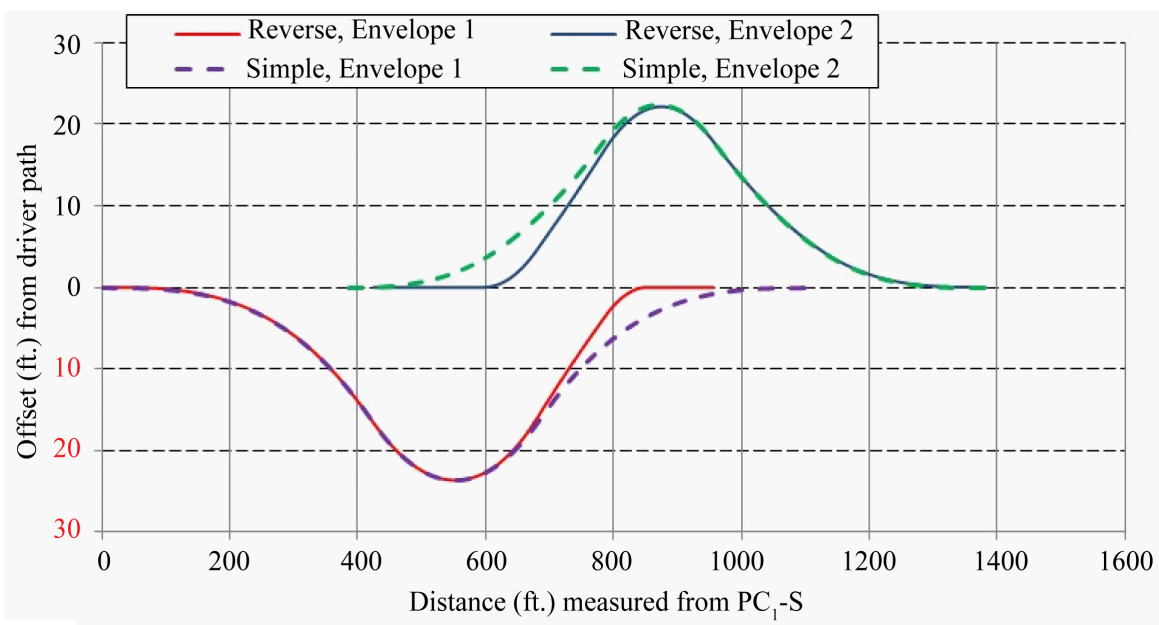

(b)

Figure 6. Comparison of simple and reverse offsets: no tangent and short tangents. (a) reverse curves with $T_{c}=0$; (b) reverse curves with $T_{c}=0.25 \mathrm{~S}$.

posed to underestimation, and third, the $5 \mathrm{ft}$ overestimation is insignificant since it is on the locations where clearance provided by lanes and shoulders $(12 \mathrm{ft}$ ) is bigger than the required $5 \mathrm{ft}$ offset.

To examine the offsets for simple curve more, a longer common tangent is tested. Figure 7(a) presents the offset lines for the curves where $T_{c}=0.5 \mathrm{~S}=212.5 \mathrm{ft}$. It is evident that there is very small discrepancy between the offset lines on small portion of the highway. For the $50 \mathrm{mph}$ reverse curve with $R_{1}=800 \mathrm{ft}$ and $R_{2}=660 \mathrm{ft}$, the minimum length of common tangent that is required for transitioning superelevation is $395 \mathrm{ft}$. The common tangent of $212.5 \mathrm{ft}$ is much smaller than the $395 \mathrm{ft}$ but still yields very small differences between offsets for isolated curves and offsets for reverse curves.

Using the $300 \mathrm{ft}$ ( $70 \%$ of $S$ ) common tangent that is recommended in the Green Book, it is found that there is no practical difference between offsets for isolated curves and reverse curves as shown in Figure 7(b). Therefore, as long as there is a reasonable length of common tangent, offset charts for simple curves may be used to determine roadside clearance for reverse curves. For reverse curves with no common tangent, a designer may use equations that have been derived in this paper or charts in Figure 8 to clear the roadside. Figure 8 (a) is for the radii ratio of $R_{1} / R_{2}=0.67$, Figure 8 (b) is for $R_{1} / R_{2}=1$, and Figure 8 (c) is for $R_{1} / R_{2}=1.5$. The radii ratios in the figure are such that the flatter radius is not more than $50 \%$ of the sharper radius. Bigger values may be unsafe.

The charts in Figure 8 imply that offsets are for curve 1 of the reverse curve or are to envelope 1 only. But they are for both curves since each direction has curve 1 . The charts are for $R / S$ ratios that are greater than 1.5 since at $R / S$ ratios greater than 1.5 offset values are not sensitive to the $R / S$ ratio [14]. To demonstrate how to use 


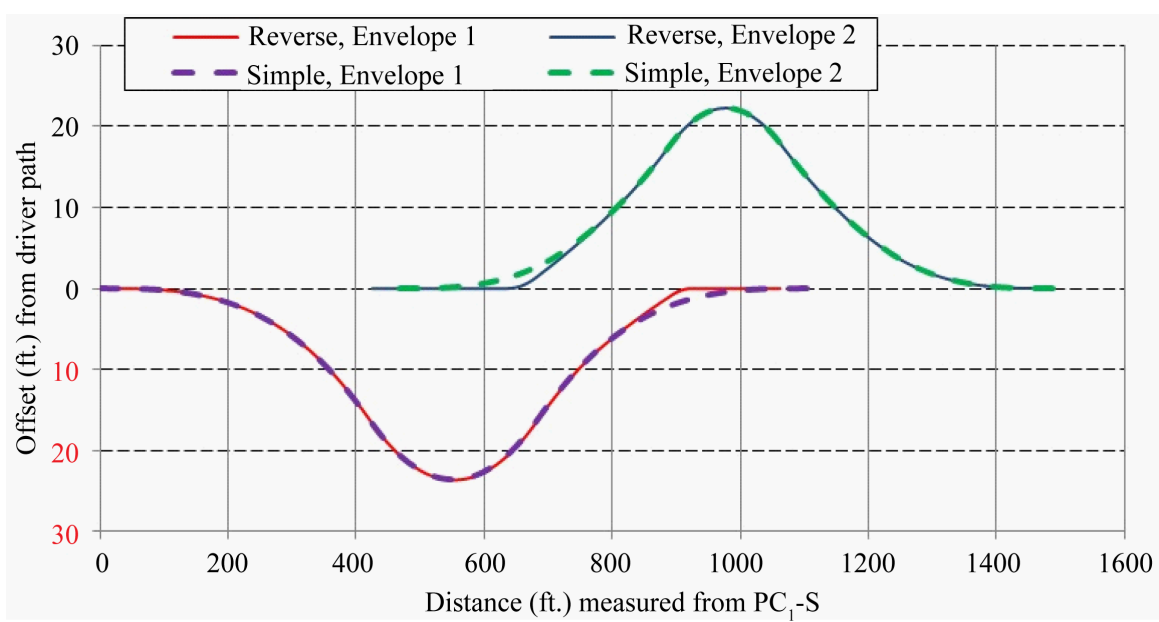

(a)

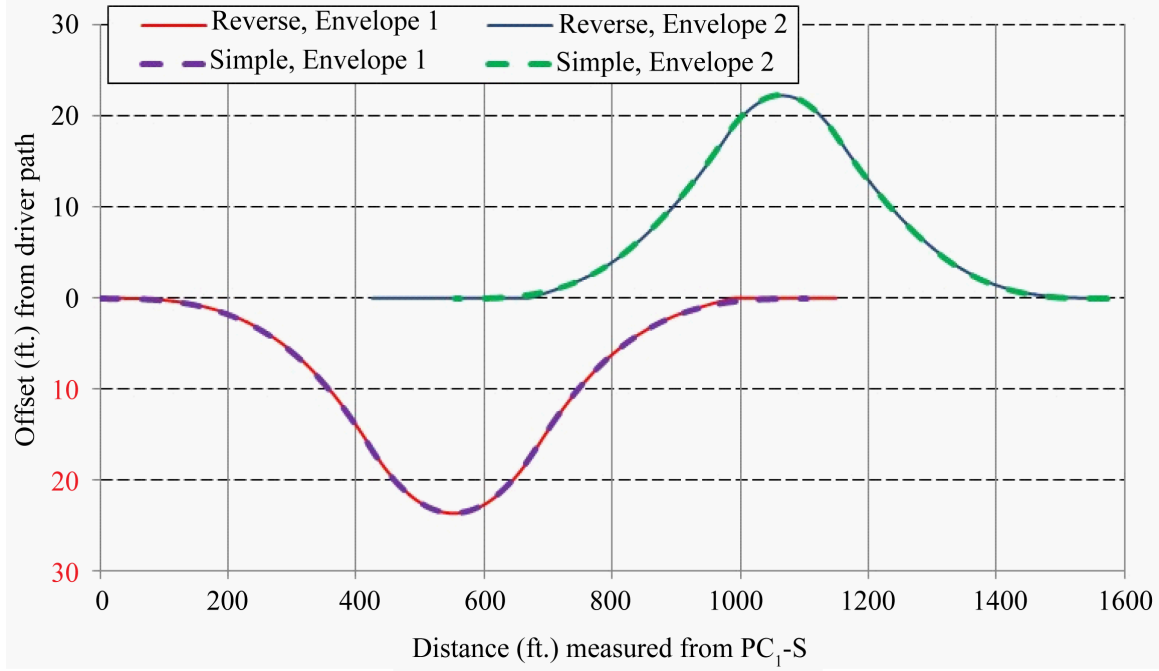

(b)

Figure 7. Comparison of simple and reverse offsets: long common tangents. (a) reverse curves with $T_{c}=0.5 S$; (b) reverse curves with $T_{c}=300 \mathrm{ft}(\approx 0.7 S)$.

the charts, consider a two lane $50 \mathrm{mph}$ highway with a reverse curve with the following features: $R_{1}=670 \mathrm{ft}, L_{1}$ $=255 \mathrm{ft}, R_{2}=1000 \mathrm{ft}, L_{2}=340 \mathrm{ft}$. Determination of offsets for the first curve follows steps below:

$R_{1} / R_{2}=670 \mathrm{ft} / 1000 \mathrm{ft}=0.67$, use Figure 8(a).

The sight distance is $425 \mathrm{ft}$ for $50 \mathrm{mph}$.

$L_{1} / S=255 / 425=0.6$, use the line labeled 0.6 in the figure.

The maximum offset in the Green Book is given by

$$
M_{\text {AASHTO }}=670[1-\cos (28.65 \times 425 / 670)]=33.42 \mathrm{ft} \text {. }
$$

For each location ratio $X / S$ (Table 1 column 3 ) an offset ratio is read on the vertical axis of Figure 8(a) as recorded in column 4 . The offset ratios are then multiplied by $M_{\text {AAsHTO }}$ of $33.42 \mathrm{ft}$ to obtain offsets (column 5).

The offsets presented in column 5 of Table 1 are only minimum values. Implementing the offsets in the field will guarantee that available sight distances are equal to the design sight distance of $425 \mathrm{ft}$. Implementing offsets that are greater than those in Table 1 will result in available sight distances that are greater than $425 \mathrm{ft}$.

\section{Summary and Conclusion}

The current Green Book and some other geometric design guidelines do not have an analytical model for clearance 

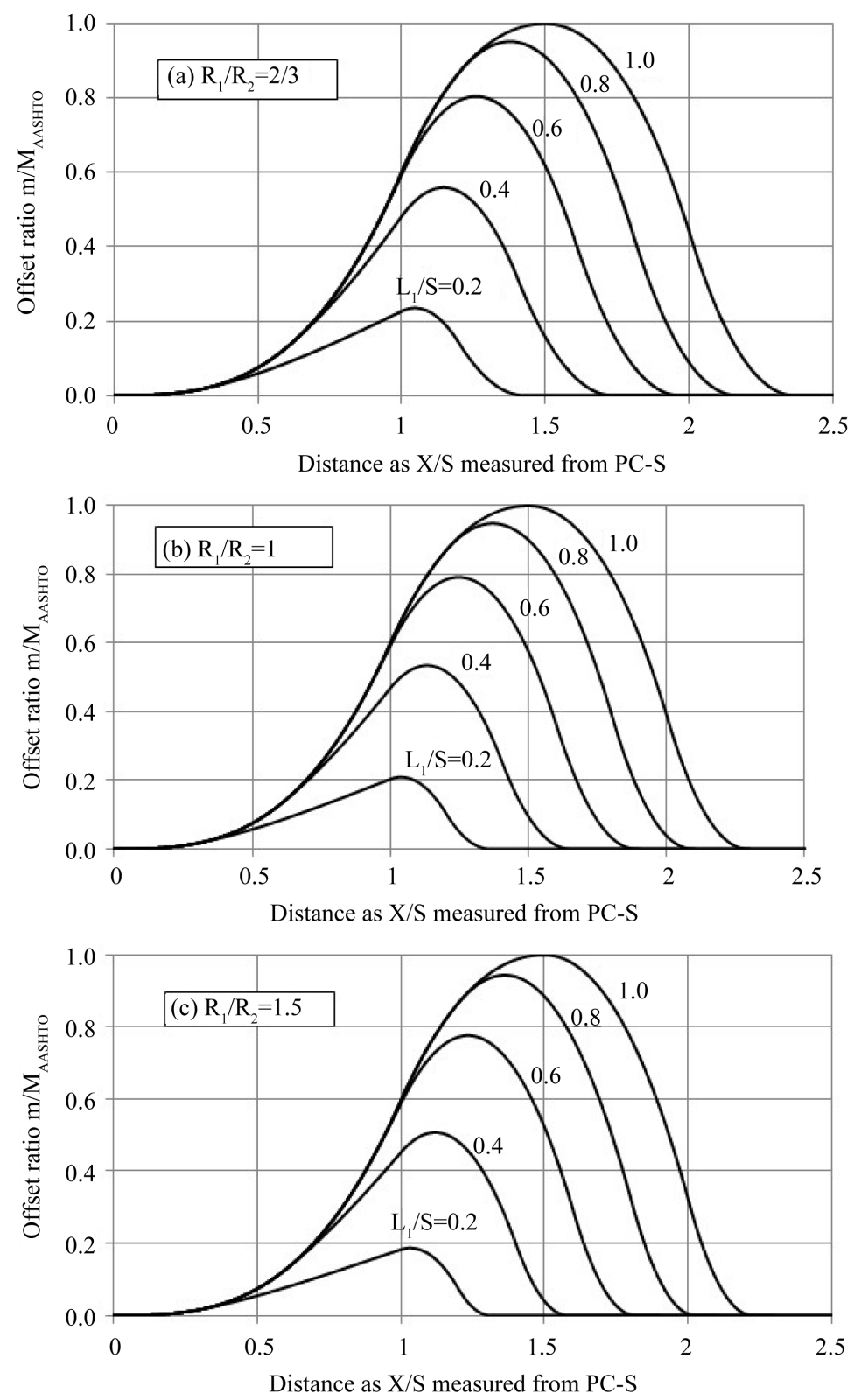

Figure 8. Design charts for reverse curves with no common tangents.

of insides of reverse curves. Applying the Green Book's model for simple curves to reverse curves will overclear sections near beginnings, ends, and near points of reverse of curvature. Over-clearance of roadsides implies extra cost of earthwork where highways pass through cut zones but it also implies unaesthetic roadsides due to extra clearance of decorative landscape features. To solve the problem, this paper developed an analytical model for clearance offsets on the inside of horizontal reverse curves. The analytical offsets were validated using offsets determined manually with the graphical method. Offsets determined with the graphical method are accurate since the method has not only been recommended in the Green Book but also has been accepted by researchers and practitioners. The only problem with the graphical method is tedium and time consumption.

The analytical model developed in this paper has many variables that make difficult development of design charts. Examination of published offset charts for isolated simple curves revealed that the charts provide a very good estimation of offsets for reverse curves if the reverse curves have common tangents whose lengths are at least $25 \%$ of design sight distance. For reverse curves with common tangents whose lengths are $50 \%$ of 
Table 1. Determination of offsets using offset charts.

\begin{tabular}{ccccc}
\hline Station & Location $X(\mathrm{ft})$ & $X / S$ & $m / M_{\text {AASHTO }}$ & Offset $(\mathrm{ft})$ \\
\hline$P C-S$ & 000 & 0.00 & 0.00 & 0.00 \\
$P C-325$ & 100 & 0.24 & 0.01 & 0.33 \\
$P C-225$ & 200 & 0.47 & 0.06 & 2.01 \\
$P C-125$ & 300 & 0.71 & 0.21 & 7.02 \\
$P C-25$ & 400 & 0.94 & 0.50 & 16.71 \\
$P C$ & 425 & 1.00 & 0.59 & 19.72 \\
$P C+75$ & 500 & 1.18 & 0.78 & 26.07 \\
$P C+110$ & 535 & 1.26 & 0.81 & 27.07 \\
$P C+175$ & 600 & 1.41 & 0.73 & 24.40 \\
$P T$ & 680 & 1.60 & 0.42 & 14.04 \\
$P T+20$ & 700 & 1.65 & 0.31 & 10.36 \\
$P T+120$ & 800 & 1.81 & 0.02 & 0.67 \\
\hline
\end{tabular}

design sight distance or those that are $300 \mathrm{ft}$ long (the $300 \mathrm{ft}$ is recommended in the Green Book), there was negligible difference between simple offsets and offsets produced for reverse curves. All lengths of common tangents analyzed were shorter than the minimum length of common tangent that was required for transitioning reversal of superelevation. Therefore, the offset charts for isolated simple curves may be used accurately for reverse curves as long as the common tangents are at least $25 \%$ of design sight distance. Use of charts for simple curves avoids development of numerous charts for reverse curves since there are many parameters involved some of which have very little impact on offsets.

Pure reverse curves are not recommended in the Green Book. Should practitioners choose to have part of their highways with pure reverse curves, the practitioners may use the derived equations or the design charts presented in Figure 8 to determine roadside clearance offsets. Practitioners may still use offsets for simple curves on pure reverse curves but it will result in over clearance at locations near points of reverse of curvature. Equations derived in this study and charts developed from those equations will find application in the areas of roadside design aiming at preserving safety and mobility of highways.

\section{Acknowledgements}

Eng. Y. Moumouni, Eng. A.J. Zegge, and Mr. A. Mnyangala thank you all.

\section{References}

[1] AASHTO (2011) A Policy on Geometric Design of Highways and Streets. American Association of State Highway and Transportation Officials, Washington DC.

[2] Raymond, W.L. (1972) Offsets to Sight Obstructions near the Ends of Horizontal Curves. ASCE Journal of Civil Engineering, 42, 71-72.

[3] Olson, P.L., Cleveland, D.E., Fancher, P.S. and Schneider, L.W. (1984) Parameters Affecting Stopping Sight Distance. Research Report NCHRP 270, Washington DC.

[4] Cleveland, D.E., Kostyniuk, L.P., Waissi, G.R., Olson, P.L. and Fancher, P.S. (1985) Stopping Sight Distance Parameters. Transportation Research Record No. 1026, Transportation Research Board of the National Academies, Washington DC, 13-23.

[5] Waissi, G.R. and Cleveland, D.E. (1987) Sight Distance Relationships Involving Horizontal Curves. Transportation Research Record No. 1122, Transportation Research Board of the National Academies, Washington DC, 96-107.

[6] Glennon, J.C. (1987) Effects of Sight Distance on Highway Safety. Transportation Research Board (TRB) State of the Art Report No. 6, Transportation Research Board Business Office, Washington DC, 64-77. 
[7] Easa, S.M. (1991) Lateral Clearance to Vision Obstacles on Horizontal Curves. Transportation Research Record No. 1303, Transportation Research Board of the National Academies, Washington DC, 22-32.

[8] Hassan, Y., Easa, S.M. and Abd El Halim, A.O. (1995) Sight Distance on Horizontal Alignments with Continuous Lateral Obstructions. Transportation Research Record No. 1500 Transportation Research Board of the National Academies, Washington DC, 31-42.

[9] Easa, S.M. (1994) Design Considerations for Highway Reverse Curves. Transportation Research Record No. 1445, Transportation Research Board of the National Academies, Washington DC, 1-11.

[10] Lovell, D.J. (1999) Automated Calculation of Sight Distance from Horizontal Geometry. Journal of Transportation Engineering, 125, 297-304. http://dx.doi.org/10.1061/(ASCE)0733-947X(1999)125:4(297)

[11] Lovell, D.J., Jong, J.C. and Chang, P.C. (2001) Improvement to Sight Distance Algorithm. Journal of Transportation Engineering, 127, 283-288. http://dx.doi.org/10.1061/(ASCE)0733-947X(2001)127:4(283)

[12] Lovell, D.J., Jong, J.C. and Chang, P.C. (2001) Clear Zone Requirements Based on Horizontal Sight Distance Considerations. Transportation Research Part A: Policy and Practice, 35, 391-411. http://dx.doi.org/10.1016/S0965-8564(99)00064-6

[13] Lovell, D.J. and Iida, T. (2003) The Exact Clear Zone Envelope for Piecewise-Linear Alignment Data. Transportation Research Part B: Methodological, 37, 485-499. http://dx.doi.org/10.1016/S0191-2615(02)00029-2

[14] Mauga, T. (2015) New Spiral Curves for Appropriate Transition of Minimum Roadside Clearance on Simple Curves. Journal of Transportation Technologies, 5, 141-158. 